\title{
Electrical properties of in situ phosphorus- and boron-doped polycrystalline SiGeC films
}

\author{
I. M. Anteney, ${ }^{\text {a) }}$ G. J. Parker, P. Ashburn, and H. A. Kemhadjian \\ Department of Electronics and Computer Science, University of Southampton, Southampton, SO17 1BJ, \\ United Kingdom
}

(Received 15 February 2000; accepted for publication 30 May 2000)

\begin{abstract}
The sheet resistance, effective carrier concentration, and Hall mobility of in situ boron- and phosphorus-doped polycrystalline $\mathrm{Si}_{0.82-y} \mathrm{Ge}_{0.18} \mathrm{C}_{y}$ films are presented for carbon contents between $0 \%$ and $4 \%$. Phosphorus and boron doping levels of $4 \times 10^{19}$ and $2 \times 10^{20} \mathrm{~cm}^{-3}$ were achieved for the $n$ - and $p$-type layers, respectively, and remained largely unaffected by carbon content. The phosphorus-doped films showed a dramatic increase in sheet resistivity and a corresponding drop in effective carrier concentration and Hall mobility. In contrast, the boron-doped films showed only a minor increase in resistivity. This is attributed to interstitial carbon increasing the defect density and also shifting the defect energy levels at the grain boundaries towards the valence band. This causes an increase in the grain-boundary energy barrier in $n$-type layers, but leaves the $p$-type layers largely unaffected. (C) 2000 American Institute of Physics. [S0003-6951(00)01730-7]
\end{abstract}

For many years, polycrystalline silicon has been a major contributor to the success of silicon integrated-circuit technology in applications such as gates for metal-oxidesemiconductor (MOS) transistors, ${ }^{1}$ polycrystalline emitters for bipolar transistors, ${ }^{2}$ and thin-film transistors for flat-panel displays. ${ }^{3}$ More recently, considerable interest has been shown in polycrystalline $\mathrm{SiGe}$ films ${ }^{4-7}$ because of lower growth temperatures, increased dopant activation, and the ability to tailor the MOS work function by adjusting the Ge concentration. Different electrical properties have been reported for $n$ - and $p$-type polycrystalline SiGe layers. In $p$-type polycrystalline $\mathrm{SiGe}$, the resistivity decreases with increasing Ge content, which has been attributed to increases in both hole mobility and dopant activation with increasing Ge incorporation. ${ }^{8,9}$ In contrast, Bang et al. ${ }^{8}$ have shown that for $n$-type films containing less than $25 \%$ Ge, the Hall mobility increases, but the effective carrier concentration steadily decreases, with increasing Ge content. The net effect is a slight decrease in the resistivity at low-Ge concentrations. For layers with Ge concentrations above 25\%, a large drop in phosphorus activation combined with a drop in the Hall mobility is observed, ${ }^{5,8}$ causing a large increase in resistivity. This was attributed to increased phosphorus segregation to the grain boundaries with increasing Ge content.

Considerable interest has been shown in single-crystal $\mathrm{SiGeC}$ for applications in heterojunction bipolar transistors. ${ }^{10}$ Polycrystalline $\mathrm{SiGeC}$ is also of interest because it offers the prospect of increased band gaps, ${ }^{11}$ and hence, would give an additional degree of freedom for bandgap engineering. Although there is a large body of work on single-crystal $\mathrm{SiGeC}$ films, little has been published on the properties of polycrystalline $\mathrm{SiGeC}$ films. In this letter we, therefore, report the electrical properties of polycrystalline $\mathrm{SiGeC}$ layers as a function of carbon content.

In situ doped $p$ - and $n$-type amorphous $\mathrm{Si}_{0.82-y} \mathrm{Ge}_{0.18} \mathrm{C}_{y}$ layers were deposited by chemical-vapor deposition at 500

${ }^{a)}$ Electronic mail: ima96r@ecs.soton.ac.uk or $540{ }^{\circ} \mathrm{C}$ on oxide-covered (100) silicon wafers. The deposition gases were $\mathrm{Si}_{2} \mathrm{H}_{6}, \mathrm{GeH}_{4}$, and $\mathrm{SiCH}_{6}$ for the $\mathrm{Si}, \mathrm{Ge}$, and $\mathrm{C}$ sources, respectively. The in situ dopant was introduced during growth using $\mathrm{PH}_{3}$ or $\mathrm{B}_{2} \mathrm{H}_{6}$ gases for the $n$ - and $p$-type sources, respectively. In all cases, the growth pressure was maintained at 4 Torr. Following deposition, the layers were capped with $200 \mathrm{~nm}$ of deposited oxide and then annealed at $1000^{\circ} \mathrm{C}$ for $30 \mathrm{~s}$. This anneal converts the amorphous $\mathrm{Si}_{0.82-y} \mathrm{Ge}_{0.18} \mathrm{C}_{y}$ into a polycrystalline material and activates the dopant introduced during growth. The oxide cap was then removed and the van der Pauw structures defined for Hall measurements.

Secondary ion mass spectroscopy analysis showed that for both the $n$ - and $p$-type layers, the doping profiles were approximately uniform, and largely unaffected by the methyl-silane flow rate. Average concentrations achieved were $4.2 \times 10^{19}$ and $2 \times 10^{20} \mathrm{~cm}^{-3}$ for the phosphorus- and boron-doped layers, respectively. In addition, it was also found that the carbon concentrations in the layers were uniform but consistently higher in the $p$-type samples. Methylsilane flows of $0-10 \mathrm{sccm}$ resulted in carbon concentrations ranging from $0 \%$ to $1.5 \%$ for phosphorus and $0 \%$ to $4 \%$ for boron-doped layers. In addition, cross-sectional transmission electron microscopy showed that the layers were polycrystalline, with an average grain size of approximately $200 \mathrm{~nm}$.

The resistivities of the $n$ - and $p$-type films as a function of $\mathrm{C}$ content are shown in Fig. 1, where it can be seen that the effect of $\mathrm{C}$ on the two films is significantly different. The $n$-type sample exhibits a dramatic increase in resistivity with $\mathrm{C}$ concentration, rising from a value of $10 \mathrm{~m} \Omega \mathrm{cm}$ with no carbon, to $2.4 \Omega \mathrm{cm}$ for $0.6 \% \mathrm{C}$. Further increase in the $\mathrm{C}$ content to $1.5 \%$ resulted in a layer that was highly resistive, even though it was highly doped. In contrast, the $p$-type sample shows only a moderate increase in resistivity, from $2.6 \mathrm{~m} \Omega \mathrm{cm}$ with no carbon to $14.7 \mathrm{~m} \Omega \mathrm{cm}$ for $4 \% \mathrm{C}$. Values of polycrystalline $\mathrm{SiGe}$ resistivity taken from the literature are also shown in Fig. 1, and indicate that the resistivities 


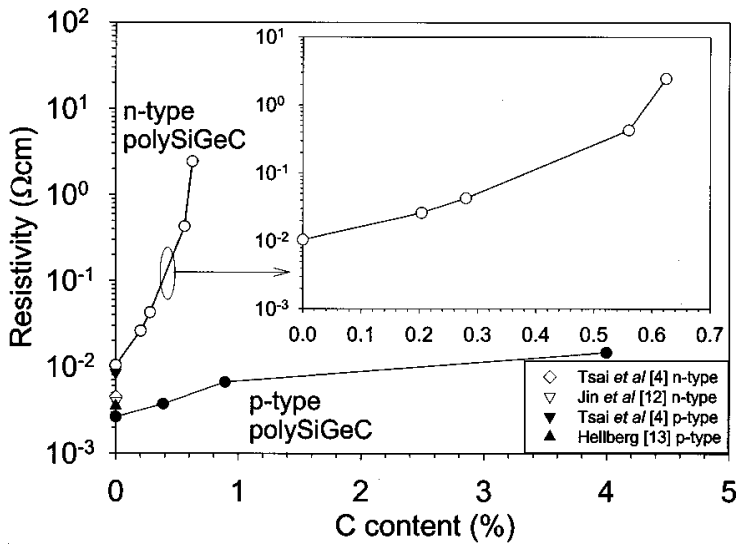

FIG. 1. Graph of layer resistivity vs carbon content for the $n$ - and $p$-type polycrystalline $\mathrm{Si}_{0.82-y} \mathrm{Ge}_{0.18} \mathrm{C}_{y}$ layers.

achieved for the layers containing no carbon are comparable to those reported. ${ }^{4,12,13}$

Figure 2 shows the effective carrier concentration as a function of carbon content. For the $p$-type samples, the effective carrier concentration decreases slowly with carbon content, dropping from $2.3 \times 10^{20} \mathrm{~cm}^{-3}$ for zero carbon to $1.19 \times 10^{20} \mathrm{~cm}^{-3}$ for $4 \% \mathrm{C}$. In contrast, the $n$-type samples exhibit a much larger reduction in carrier concentration, falling from $2.89 \times 10^{19} \mathrm{~cm}^{-3}$ for no carbon to 7.36 $\times 10^{18} \mathrm{~cm}^{-3}$ for a layer containing $0.5 \%$ C. No meaningful value was obtainable for the layer containing $0.6 \% \mathrm{C}$.

Figure 3 shows plots of the Hall mobility in the $n$ - and $p$-type samples as a function of $\mathrm{C}$ content. For the $n$-type samples, the addition of carbon causes a dramatic decrease in electron mobility from a value of $21 \mathrm{~cm}^{2} / \mathrm{V}$ s with no carbon added to just above $2 \mathrm{~cm}^{2} / \mathrm{V}$ s for the layer containing $0.5 \%$ carbon. Once again, the effect of $\mathrm{C}$ on the $p$-type mobility is much less severe, decreasing from $10 \mathrm{~cm}^{2} / \mathrm{V}$ s for no carbon to $3.4 \mathrm{~cm}^{2} / \mathrm{V} \mathrm{s}$ for $4 \% \mathrm{C}$.

The above results indicate that the effect of carbon on the electrical properties of polycrystalline SiGe layers is significantly different in $n$ - and $p$-type layers. In P-doped layers, the resistivity dramatically increases with $\mathrm{C}$ concentration, whereas in B-doped layers the increase in resistivity is much smaller. To give insight into this behavior, models for

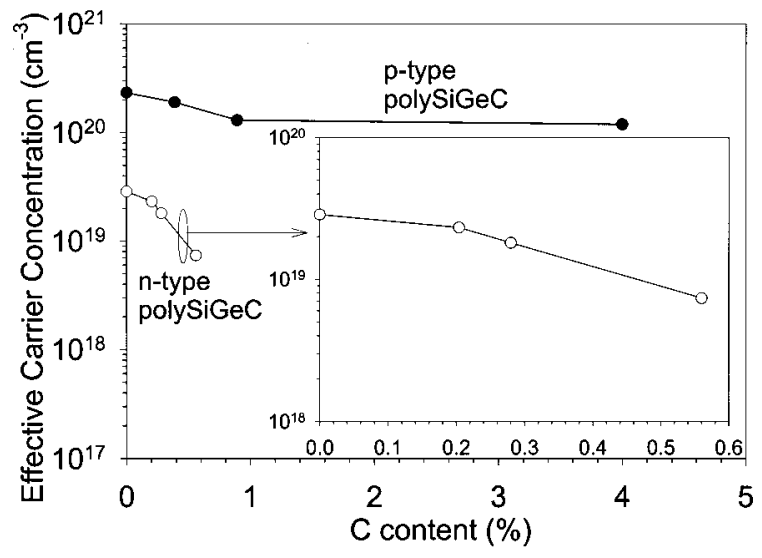

FIG. 2. Graph of effective carrier concentration vs carbon content for the $n$ and $p$-type polycrystalline $\mathrm{Si}_{0.82-y} \mathrm{Ge}_{0.18} \mathrm{C}_{y}$ layers. p-type polycrystalline $\mathrm{Si}_{0.82-y} \mathrm{Ge}_{0.18} \mathrm{C}_{y}$ layers. $\quad$ boundaries in polycrystalline $\mathrm{SiGeC}$ would give rise to an
Downloaded 05 Jan 2004 to 152.78 .67 .91 . Redistribution subject to AIP license or copyright, see http://ojps.aip.org/aplo/aplcr.jsp

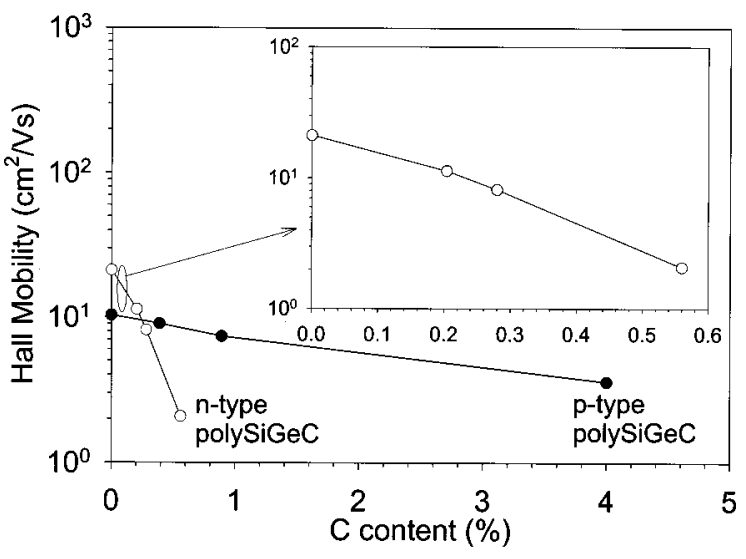

FIG. 3. Graph of Hall mobility vs carbon content for the $n$ - and $p$-type polycrystalline $\mathrm{Si}_{0.82-y} \mathrm{Ge}_{0.18} \mathrm{C}_{y}$ layers.

conduction in polycrystalline $\mathrm{Si}$ and SiGe must first be considered.

In polycrystalline silicon, defects at the grain boundaries introduce trapping centers located at energy levels near the middle of the energy gap. These traps immobilize free carriers from substitutional dopant atoms causing the defects to become electrically charged. This gives rise to a potential energy barrier that impedes the flow of carriers from one crystallite to another, thus limiting conduction. Since the defects are located around the middle of the energy gap, the effects are similar for both $n$ - and $p$-type dopants. At high dopant concentrations, the traps become fully occupied, allowing any additional free carriers to form a neutral region within the crystallite, reducing the depletion regions at the grain boundaries and lowering the potential barrier. ${ }^{14,15} \mathrm{Al}-$ though the grain-boundary barriers are similar for $n$ - and $p$-type layers, dopant segregation can result in differences in electrical behavior. Mandurah, Saraswat, and Helms ${ }^{16}$ have shown that As and $\mathrm{P}$ segregate to grain boundaries whereas $\mathrm{B}$ does not. This can result in differences in conduction since segregated dopant is electrically inactive, and hence, cannot reduce the grain-boundary energy barrier.

In polycrystalline $\mathrm{SiGe}$, the electrical behavior is different in $n$ - and $p$-type materials because the addition of $\mathrm{Ge}$ causes the trap energy levels at the grain boundaries to move towards the valence band. For $n$-type layers, this shift in energy levels results in an increase in the potential-energy barrier at the grain boundary, thus reducing the mobility within the layer for Ge concentrations above $35 \% .^{9,12}$ In addition, Bang et al. have reported a decrease in the effective carrier concentration with increasing Ge content, suggesting that Ge could also give increased phosphorus segregation. In boron-doped layers, the shift in trap energy levels towards the valence band reduces the energy barrier, and since B does not segregate to the grain boundaries, the addition of $\mathrm{Ge}$ to the polycrystalline layer increases both mobility and effective carrier concentration. ${ }^{9,12,17}$

The results obtained in this work could, therefore, be explained by the influence of $\mathrm{C}$ on the trap density and energy barrier at the grain boundaries. Londos ${ }^{18}$ has shown that interstitial $\mathrm{C}$ introduces a deep-level donor trap into the band gap of single-crystal silicon at an energy of $E_{v}+0.28 \mathrm{eV}$. It is, therefore, likely that the presence of carbon at grain 
increased trap density and a shift in trap energy towards the valence band. This shift in the trap energy level would lead to an increase in the energy barrier for $n$-type layers and a decrease for $p$ type.

In summary, for $n$-type $\mathrm{SiGeC}$ layers, the increase in energy barrier would be expected to combine with the increase in grain-boundary trap density to give a dramatic increase in resistivity. This problem is further compounded by the tendency of phosphorus to segregate to the grain boundaries, leaving less electrically active dopant to compensate these additional traps. In contrast, for $p$-type $\mathrm{SiGeC}$ layers, the increase in grain-boundary trap density is compensated by the decrease in the energy barrier, leading to a much smaller increase in the resistivity.

${ }^{1}$ T. Yamamoto, K. Uwasawa, and T. Mogamu, IEEE Trans. Electron Devices 46, 921 (1999).

${ }^{2}$ I. R. C. Post, P. Ashburn, and G. R. Wolstenholme, IEEE Trans. Electron Devices 39, 1717 (1992).

${ }^{3}$ L. Pichon, F. Raoult, K. Mourgues, K. Kission, T. Mohammed Brahim, and O. Bonnaud, Thin Solid Films 296, 136 (1997).

${ }^{4}$ J. Tsai, A. Tang, T. Noguchi, and R. Reif, J. Electrochem. Soc. 142, 3220 (1995).
${ }^{5}$ T. J. King and K. Saraswat, J. Electrochem. Soc. 141, 2235 (1994).

${ }^{6}$ V. Li, M. R. Mirabedini, R. T. Kuehn, J. J. Wortman, M. Ozturk, D. Batchelor, K. Christensen, and D. Maher, Appl. Phys. Lett. 71, 3388 (1997).

${ }^{7}$ V. Li, M. R. Mirabedini, B. Hornung, H. Heinisch, M. Xu, D. Batchelor, D. Maher, J. J. Wortman, and R. T. Kuehn, J. Appl. Phys. 83, 5469 (1998)

${ }^{8}$ D. Bang, M. Cao, A. Wang, K. Saraswat, and T. King, Appl. Phys. Lett. 66, 195 (1997).

${ }^{9}$ C. Salm, D. van Veen, D. Gravesteijn, J. Holleman, and P. Woerlee, J. Electrochem. Soc. 144, 3665 (1997).

${ }^{10}$ I. M. Anteney, G. Lippert, P. Ashburn, H. J. Osten, B. Heinemann, G. J. Parker, and D. Knoll, IEEE Trans. Electron Devices 20, 116 (1999).

${ }^{11}$ L. D. Lanzerotti, A. St. Amour, C. W. Liu, J. C. Sturm, J. K. Watanabe, and N. D. Theodore, IEEE Electron Device Lett. 17, 334 (1996).

${ }^{12}$ Z. Jin, B. Gururaj, W. Yeung, H. Kwok, and M. Wong, IEEE Trans. Electron Devices 44, 1958 (1997).

${ }^{13}$ P. Hellberg, A. Gagnor, S. Zhang, and C. S. Petersson, J. Electrochem. Soc. 144, 3968 (1997).

${ }^{14}$ T. Kamins, J. Appl. Phys. 42, 4357 (1971).

${ }^{15}$ J. Seto, J. Appl. Phys. 46, 5247 (1975).

${ }^{16}$ M. M. Mandurah, K. C. Saraswat, and C. R. Helms, J. Appl. Phys. 51, 5755 (1980).

${ }^{17}$ T. J. King, J. McVittie, K. Saraswat, and J. Pfiester, IEEE Trans. Electron Devices 41, 228 (1994).

${ }^{18}$ C. Londos, Phys. Rev. B 35, 6295 (1987). 